Chang Hyung Hong,

$\mathrm{MD}, \mathrm{PhD}$

Cherie Falvey, MPH

Tamara B. Harris, MD

Eleanor M. Simonsick,

$\mathrm{PhD}$

Suzanne Satterfield, MD,

$\mathrm{DrPh}$

Luigi Ferrucci, MD, PhD

Andrea L. Metti, MPH

Kushang V. Patel, PhD,

$\mathrm{MPH}$

Kristine Yaffe, MD

Correspondence to

Dr. Yaffe:

kristine.yaffe@ucsf.edu

\section{Anemia and risk of dementia in older adults}

\author{
Findings from the Health $\mathrm{ABC}$ study
}

ABSTRACT

Objective: To determine whether anemia is associated with incident dementia in older adults.

Methods: We studied 2,552 older adults (mean age 76.1 years; 38.9\% black; 51.8\% female) participating in the Health, Aging, and Body Composition study and free of dementia at baseline. We defined anemia using WHO criteria (hemoglobin concentration $<13 \mathrm{~g} / \mathrm{dL}$ for men and $<12 \mathrm{~g} /$ $\mathrm{dL}$ for women). Dementia diagnosis was determined by dementia medication use, hospital records, or a change in Modified Mini-Mental State (3MS) score of more than 1.5 SD from mean. Discrete time Cox proportional hazard regression models were used to examine the hazard for developing dementia associated with anemia.

Results: Of 2,552 participants, 392 (15.4\%) older adults had anemia at baseline. Over 11 years of follow-up, 455 (17.8\%) participants developed dementia. In the unadjusted model, those with baseline anemia had an increased risk of dementia (23\% vs $17 \%$, hazard ratio $=1.64 ; 95 \%$ confidence interval 1.30, 2.07) compared to those without anemia. The association remained significant after adjusting for demographics, APOE $\varepsilon 4$, baseline $3 \mathrm{MS}$ score, comorbidities, and renal function. Additional adjustment for other anemia measures (mean corpuscular volume, red cell distribution width), erythropoietin, and C-reactive protein did not appreciably change the results. There was no interaction by sex and race on risk of developing dementia.

Conclusion: Among older adults, anemia is associated with an increased risk of developing dementia. Findings suggest that further study of anemia as a risk factor for dementia and a target for intervention for cognitive health is warranted. Neurology ${ }^{\circledR}$ 2013;81:528-533

\section{GLOSSARY}

$\mathbf{3 M S}=$ Modified Mini-Mental State; $\mathbf{C l}=$ confidence interval; $\mathbf{C R P}=$ C-reactive protein; $\mathbf{D M}=$ diabetes mellitus; $\mathbf{G F R}=$ glomerular filtration rate; Health ABC = Health, Aging, and Body Composition study; HR = hazard ratio; HTN = hypertension; $\mathbf{M C V}=$ mean corpuscular volume; $\mathbf{M I}=$ myocardial infarct; $\mathbf{R D W}=$ red cell distribution width; $\mathbf{R E A L M}=$ Rapid Estimate of Adult Literacy in Medicine.

Anemia is common in community-dwelling adults aged 65 years and older, with prevalence estimates between $9.2 \%$ and $23.9 \%$ in the United States when WHO criteria are applied. ${ }^{1-5}$ In older adults, anemia is associated with morbidity and mortality, ${ }^{6,7}$ and a few recent studies suggest that anemia or abnormal hemoglobin concentrations are associated with an increased risk for dementia and rapid cognitive decline among the elderly. ${ }^{8-17}$ However, evidence from previous studies is inconclusive, in part due to methodologic limitations including cross-sectional design, which does not allow for assessment of etiologic associations. ${ }^{12-14,16}$ Moreover, the few existing longitudinal studies rely on relatively short follow-up (average approximately 3 years) ${ }^{9,15}$ and have limited generalizability (e.g., measuring cognitive function only in women ${ }^{8,11}$ or lacking racial or ethnic diversity). Prior studies are also limited in adjustment of potential confounders such as stroke, cardiovascular risk factors, erythropoietin, mean corpuscular volume (MCV), and red cell

From the Department of Psychiatry and Institute on Aging (C.H.H.), Ajou University School of Medicine, Suwon, Republic of Korea; Departments of Psychiatry (C.F., K.Y.), Epidemiology and Biostatistics (K.Y.), and Neurology (K.Y.), School of Medicine, University of California, San Francisco; San Francisco Veterans Affairs Medical Center (C.F., K.Y.), San Francisco; Laboratory of Epidemiology, Demography, and Biometry, Intramural Research Program (T.B.H.), and Clinical Research Branch (E.M.S., L.F.), National Institute on Aging, Baltimore, MD; Department of Preventive Medicine (S.S.), University of Tennessee Health Science Center, Memphis; Department of Epidemiology (A.L.M.), Graduate School of Public Health, University of Pittsburgh, PA; and Department of Anesthesiology and Pain Medicine (K.V.P.), University of Washington, Seattle.

Go to Neurology.org for full disclosures. Funding information and disclosures deemed relevant by the authors, if any, are provided at the end of the article. 
distribution width (RDW) that might be associated with the mechanism linking anemia to incident dementia.

We sought to determine the association between anemia and risk of dementia in the prospective Health, Aging, and Body Composition (Health $A B C$ ) study. The Health $A B C$ study consists of a relatively large and diverse cohort followed over 11 years with broad assessment of risk factors that allows us to evaluate the relationship between anemia and incident dementia while adjusting for numerous potential confounders, including those that have previously been reported to be related to dementia and anemia.

METHODS Study population. Participants were enrolled in the ongoing Health ABC study beginning in 1997, a prospective cohort of 3,075 community-dwelling white and black older adults aged 70-79 years and living in Memphis, Tennessee, or Pittsburgh, Pennsylvania. To identify potential participants, a random sample of white and all of the black Medicare-eligible elders within designated zip code areas were designated as potential participants. Exclusion criteria included reported difficulties performing mobility-related activities of daily living or walking a quarter of a mile or climbing 10 steps without resting. In year 3 of the study (19992000), blood samples were obtained on 2,552 participants that included measures of anemia. This first anemia assessment is considered the baseline for our analysis.

Standard protocol approvals, registrations, and patient consents. This study was approved by the institutional review boards of the University of Pittsburgh and the University of Tennessee, Memphis, and that of the coordinating center, the University of California, San Francisco. All participants signed an informed written consent, approved by the institutional review boards at the clinical sites.

Primary measurements. Erythropoietin and anemia measures such as hemoglobin concentrations, MCV, and RDW were measured at baseline. Anemia was defined using the WHO criteria of hemoglobin concentrations below $12 \mathrm{~g} / \mathrm{dL}$ for women and below $13 \mathrm{~g} / \mathrm{dL}$ for men. ${ }^{18}$

Cognitive function was assessed at years 1, 3, 5, 8, 10, and 11 with a global test, the Modified Mini-Mental State (3MS) examination. ${ }^{19}$ Incident dementia was determined by prescribed dementia medications (i.e., galantamine, rivastigmine, memantine, donepezil, and tacrine), hospital records, or a race-stratified decline in 3MS score of more than 1.5 SD from baseline mean.

Covariates Possible covariates included baseline self-reported age, race, sex, and education (categorized as < high school, high school, or $>$ high school). Literacy was measured with the Rapid Estimate of Adult Literacy in Medicine (REALM) test. ${ }^{20}$ REALM scores were categorized by reading level ( $0-6$ th, 7 th- 8 th, and $\geq 9$ th grade). ${ }^{21}$ Depressive symptoms were assessed using the 20-item Center for Epidemiologic Studies-Depression Scale. ${ }^{22}$ Body mass index $\left(\mathrm{kg} / \mathrm{m}^{2}\right)$ was calculated from direct height and weight measurements at baseline. Hypertension (HTN) and diabetes mellitus (DM) were determined using self-report, medication use, and verification of hospital records by an adjudicator according to prescribed algorithms. History of stroke and myocardial infarct (MI) were based on self-report, clinic data, and medication use. Glomerular filtration rate (GFR) was estimated at baseline using the Cockcroft-Gault equation. ${ }^{23} A P O E$ genotype was determined by the $5^{\prime}$-nuclease assay ${ }^{24}$ in the Human Genetics laboratory at the University of Pittsburgh and participants were coded as $\varepsilon 4$ carriers or noncarriers. Serum concentrations of C-reactive protein (CRP) were measured in duplicate by ELISA on the basis of purified protein and polyclonal anti-CRP antibodies.

Statistical analyses. We first performed bivariate analyses to investigate baseline demographic and health-related characteristics between the group with anemia and the group without anemia. We used $\chi^{2}$ analysis for categorical variables and $F$ test for continuous variables. Time to dementia was coded as first record of diagnosis or censored from observation at the end of the period for which data were available. We used Kaplan-Meier survival curves to plot the survival curve for incident dementia by presence of anemia. Discrete time Cox proportional hazard regression models were used to examine the hazard for developing dementia associated with anemia.

To investigate whether other concurrent conditions could explain the association, we assessed the relationship within 4 different models. Model 1 made adjustments for age, race, sex, and education. Model 2 adjusted for APOE \&4, 3MS score, and all factors adjusted in model 1. Model 3 added comorbidities (stroke, HTN, $\mathrm{DM}$, and $\mathrm{MI}$ ), renal function (GFR), and all factors adjusted in model 2. Finally, model 4 included other anemia measures (MCV, $\mathrm{RDW})$, erythropoietin, CRP, literacy, and all factors adjusted for in model 3. We also tested for an interaction between race and anemia, and in a sensitivity analysis, we evaluated the association between anemia and change in $3 \mathrm{MS}$ over the follow-up period using linear mixed-effects models. All analyses were conducted using SAS statistical software, version 9.2 (SAS Institute Inc, Cary, NC), and were 2-tailed with the statistical significance level set at $p<0.05$.

RESULTS Of 2,552 participants, 393 (15.4\%) older adults had anemia at baseline. Mean age was 76.1 (SD 2.8) years, 1,322 (51.8\%) were female, and 994 (38.9\%) were black. Over the 11 years of follow-up, 455 (17.8\%) participants developed incident dementia. Participants with anemia were more likely to be older, black, and male, and have an APOE $\& 4$ allele, less education, lower REALM and 3MS score, and history of HTN, DM, and MI ( $p<0.05$ for all). Participants with anemia also had higher levels of RDW, erythropoietin, and CRP, and lower levels of MCV and GFR ( $p<0.05$ for all) (table 1).

Older adults who had anemia at baseline were more likely to develop dementia $(n=89,22.7 \%)$ compared to those who did not have anemia $(\mathrm{n}=366,17.0 \%)$ ( $p=0.007$ ) (figure). In the unadjusted Cox proportional hazard models, those with baseline anemia had an increased risk of dementia (unadjusted hazard ratio $[\mathrm{HR}]=1.64 ; 95 \%$ confidence interval $[\mathrm{CI}] 1.30$, 2.07) compared to those without anemia. Table 2 shows the HR of dementia for subjects with anemia after adjustment for different potential confounders. The direction and strength of the association between anemia and incident dementia remained even after adjustment for potential confounders including those previously associated with anemia and dementia (models 1-4). After adjusting for age, race, sex, education, literacy, $A P O E \varepsilon 4$ status, $3 \mathrm{MS}$ score, comorbidities 
Table 1 Baseline characteristics by anemia among the 2,552 participants ${ }^{a}$

\begin{tabular}{|c|c|c|c|}
\hline Characteristics & Anemia $^{b}(n=393)$ & No anemia $(n=2,159)$ & $p$ Value \\
\hline Age, y & 76.5 (2.9) & $76.0(2.8)$ & 0.002 \\
\hline Black & $228(58.0)$ & 766 (35.5) & $<0.0001$ \\
\hline Female & $171(43.5)$ & $1,151(53.3)$ & 0.0003 \\
\hline Education $<$ high school & $128(32.7)$ & $463(21.5)$ & $<0.0001$ \\
\hline$A P O E \varepsilon 4$ & 122 (32.3) & $558(27.4)$ & 0.05 \\
\hline 3MS score & $87.5(9.01)$ & 90.7 (8.05) & $<0.0001$ \\
\hline Stroke & $35(8.9)$ & $173(8.0)$ & 0.55 \\
\hline Myocardial infarction & 69 (17.6) & $277(12.8)$ & 0.01 \\
\hline Hypertension & $258(65.7)$ & $1,293(59.9)$ & 0.03 \\
\hline Diabetes & $120(30.6)$ & 507 (23.5) & 0.003 \\
\hline Glomerular filtration rate, $\mathrm{mL} / \mathrm{min}$ & $63.0(22.83)$ & $71.4(20.30)$ & $<0.0001$ \\
\hline Depression & $19(4.8)$ & $80(3.7)$ & 0.29 \\
\hline Body mass index, $\mathrm{kg} / \mathrm{m}^{2}$ & $27.2(4.7)$ & $27.3(5.3)$ & 0.73 \\
\hline Mean corpuscular volume, $\mathrm{fL}$ & $88.2(7.04)$ & $91.1(4.90)$ & $<0.0001$ \\
\hline Red cell distribution width, \% & $14.3(1.98)$ & $13.4(1.04)$ & $<0.0001$ \\
\hline Erythropoietin, $\mathrm{mlU} / \mathrm{mL}$ & $21.1(16.15)$ & $13.2(6.87)$ & $<0.0001$ \\
\hline C-reactive protein, $\mu \mathrm{g} / \mathrm{mL}$ & $3.4(4.7)$ & $2.8(4.5)$ & 0.02 \\
\hline REALM score $\leq 6$ th grade & $83(21.1)$ & $232(10.7)$ & $<0.0001$ \\
\hline
\end{tabular}

Abbreviations: $3 M S$ = Modified Mini-Mental State; REALM = Rapid Estimate of Adult Literacy in Medicine.

${ }^{a}$ Values are mean (SD) or $\mathrm{n}(\%)$.

${ }^{\mathrm{b}}$ Anemia was defined using the WHO criteria (hemoglobin concentrations below $12 \mathrm{~g} / \mathrm{dL}$ for women and below $13 \mathrm{~g} / \mathrm{dL}$ for men).

(stroke, HTN, DM, and MI), renal function (GFR), anemia measures (MCV, RDW), erythropoietin, and CRP, the results remained statistically significant (adjusted HR $=1.49$; 95\% CI 1.11, 2.00) (table 2). When hemoglobin level was considered as a continuous variable in the fully adjusted models, the association was also significant $(\beta=-0.12, p$ value $=0.02)$. There were no interactions between anemia and sex or race on risk of developing dementia $(p>$ 0.10 for both).

In a sensitivity analysis, we evaluated the relationship between anemia and change in cognition over

Figure Kaplan-Meier survival function of time to dementia diagnosis by baseline anemia

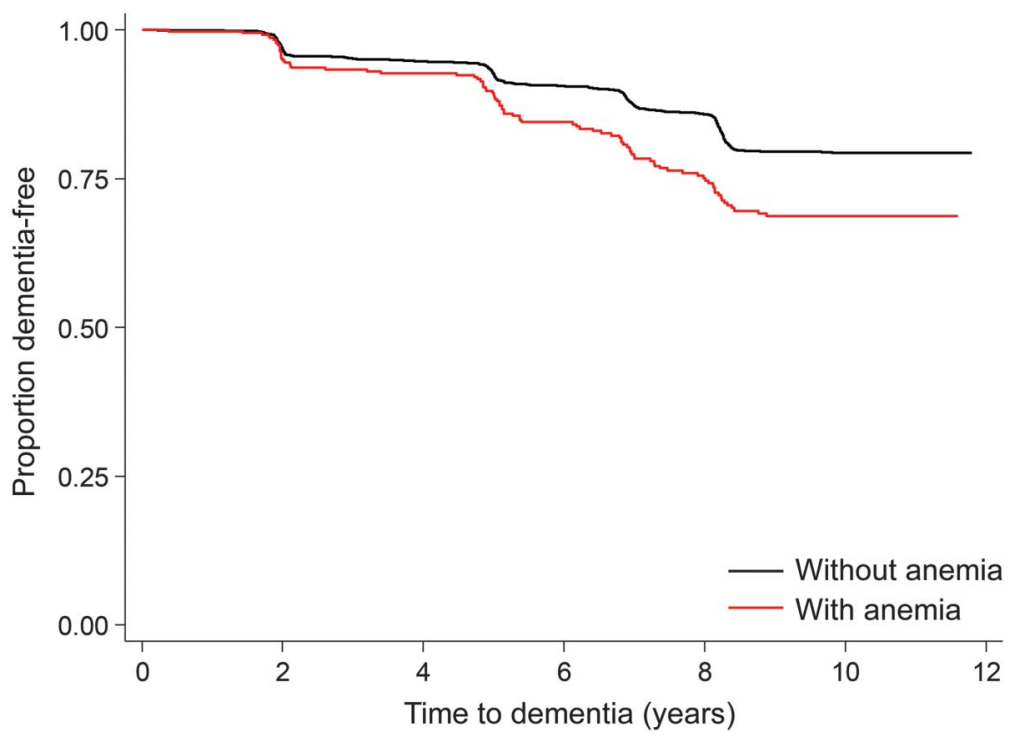

Anemia was defined using the WHO criteria (hemoglobin concentrations below $12 \mathrm{~g} / \mathrm{dL}$ for women and below $13 \mathrm{~g} / \mathrm{dL}$ for men). 
Table 2 Cox proportional hazard ratios for time to dementia by baseline anemia ${ }^{a}$

$\begin{array}{lr}\text { Model } & \text { HR (95\% Cl) } \\ \text { Unadjusted } & 1.64(1.30,2.07) \\ \text { Model 1: demographics (age, sex, education, and race) } & 1.41(1.12,1.80) \\ \text { Model 2: model } 1+\text { APOE } \varepsilon 4 \text { and 3MS score } & 1.47(1.14,1.88) \\ \text { Model 3: model } 2+\text { comorbidities (stroke, HTN, DM, and MI) and renal function (GFR) } & 1.39(1.07,1.80)\end{array}$

Model 4: model 3 + anemia measures (MCV and RDW), erythropoietin, C-reactive protein, and literacy 1.49 (1.11, 2.00$)$

Abbreviations: $3 \mathrm{MS}=$ Modified Mini-Mental State; $\mathrm{Cl}=$ confidence interval; DM = diabetes mellitus; GFR = glomerular filtration rate; HR = hazard ratio; $\mathrm{HTN}=$ hypertension; $\mathrm{MCV}=$ mean corpuscular volume; $\mathrm{Ml}=$ myocardial infarction; $\mathrm{RDW}=$ red cell distribution width.

${ }^{a}$ Anemia was defined using the WHO criteria (hemoglobin concentrations below $12 \mathrm{~g} / \mathrm{dL}$ for women and below $13 \mathrm{~g} / \mathrm{dL}$ for men).

time. The results were consistent with our survival models. Anemia was significantly associated with decline in 3MS ( $\beta=-0.19, p=0.02)$.

DISCUSSION In this prospective study of 2,552 older adults without baseline dementia, participants with anemia had a $41 \%$ higher risk of developing dementia over 11 years than those without anemia after adjusting for age, race, sex, and education. The association was still substantial and significant after adjusting for potential confounders, including comorbidities and other markers of red cell health.

These results are consistent with findings from 2 prior prospective cohort studies. One found that anemia was associated with a 2-fold increased hazard for developing dementia over 3 years among 1,139 older adults. ${ }^{9}$ The other study found participants with anemia had a $60 \%$ increased hazard for developing $\mathrm{Alz}_{\text {- }}$ heimer disease dementia over 3.3 years among 881 older adults. ${ }^{15}$ Our study provides evidence that anemia may be an important risk factor for developing dementia. In comparison to the other studies, our study had a larger sample size, much longer follow-up, and a diverse population, and while there was not a significant interaction between race and anemia, it is important to note the higher burden of anemia among black subjects as this could be one factor associated with the higher burden of dementia in this group.

The mechanisms linking anemia to incident dementia are not clearly understood, although 4 hypotheses have been previously proposed. First, the chronic brain hypoxia associated with anemia may contribute to risk of dementia. ${ }^{9}$ Anemia has been associated with progression of white matter disease in older adults with high blood pressure. ${ }^{25} \mathrm{~A}$ meta-analysis of population-based studies yielded a significant association between white matter hyperintensities and occurrence of all types of dementia. ${ }^{26}$ However, our results were robust to adjustment for stroke and other cardiovascular risk factors. Second, anemia due to chronic kidney disease may be associated with dementia. Besides regulating red blood cell production, erythropoietin receptors have been localized in the brain ${ }^{27}$ and seem to have a protective effect in animal models of stroke and hypoxia, ${ }^{28,29}$ and lower erythropoietin levels may increase the risk of neuronal degeneration. Animal studies have also found that erythropoietin reduces tau phosphorylation ${ }^{29}$ and protects against $\beta$-amyloid-induced cell death. ${ }^{30,31}$ In our study, adjustment for serum erythropoietin as well as for kidney function did not attenuate the results; however, blood level measures may not reflect CNS penetration. Third, anemia due to deficiency of micronutrients such as iron and vitamin B12 may also be associated with cognitive impairment and dementia. Deficiency of iron, because it plays an important role in oxygen transport and storage in the brain, may lead to cerebral hypoxia and cognitive decline. ${ }^{32}$ In addition, vitamin B12 deficiency has been associated with Alzheimer disease dementia and vascular dementia. ${ }^{33}$ Given that iron and vitamin B12 deficiency anemia are microcytic and macrocytic anemia respectively and that adjustment for MCV and RDW did not change our results, this pathway is less likely. RDW can capture iron deficiency early before MCV changes and help adjust for the presence of multiple nutritional deficiencies that would not change the MCV. Finally, it is also possible that anemia is a marker of poor health as other studies have demonstrated that dementia is often associated with a range of age-related health outcomes, ${ }^{34}$ or there may be another latent variable associated with both anemia and dementia that is driving this association; thus, the implications of these findings for dementia prevention are not clear.

Our study has several strengths, including a prospective design with a long follow-up period and a relatively large sample of community-dwelling older adults without dementia at baseline. We were able to adjust for a broad range of potential confounders, including those that have previously been shown to be related to dementia and anemia. In particular we adjusted for CRP to potentially rule out anemia of chronic disease as a contributor to dementia and adjusted for literacy and $A P O E$ to control for the rate of cognitive decline modified by cognitive reserve. There are also several limitations that 
should be taken into consideration when interpreting these results. Dementia diagnosis in our study may be less sensitive than diagnosis using structured clinical interview. We also did not have information on type of dementia and anemia.

Our findings support the hypothesis that anemia may be a risk factor for incident dementia over time in a cohort of dementia-free older adults, but in order to further determine whether prevention strategies for dementia should especially target hemoglobin levels in older adults or should instead focus on improving general health status, additional studies are needed to establish the mechanisms of this association.

\section{AUTHOR CONTRIBUTIONS}

Dr. Hong: study concept and design, analysis and interpretation, drafting and critical revision of the manuscript. C. Falvey: drafting and critical revision of the manuscript. Dr. Harris: critical revision of the manuscript. Dr. Simonsick: critical revision of the manuscript. Dr. Satterfield: critical revision of the manuscript. Dr. Ferrucci: critical revision of the manuscript. A.L. Metti: critical revision of the manuscript. Dr. Patel: critical revision of the manuscript. Dr. Yaffe: study concept and design, acquisition of data, analysis and interpretation, drafting and critical revision of the manuscript, study supervision.

\section{STUDY FUNDING}

NIA contract numbers: N01-AG-6-2101, N01-AG-6-2103, N01-AG-62106. NIA grant numbers: R01-AG028050, NINR grant R01NR012459. This research was supported in part by the Intramural Research Program of the NIH and National Institute of Aging. Dr. Yaffe is supported in part by a National Institute of Aging Grant (K24AG031155) and a grant from the American Health Assistance Foundation (A201-0029).

\section{DISCLOSURE}

C.H. Hong, C. Falvey, T. Harris, E. Simonsick, S. Satterfield, L. Ferrucci, A. Metti, and K. Patel report no disclosures. K. Yaffe has served on data safety monitoring boards for Takeda, Inc., and the NIH and has served as a consultant for Novartis, Inc. Go to Neurology.org for full disclosures.

Received March 4, 2013. Accepted in final form April 18, 2013.

\section{REFERENCES}

1. Denny SD, Kuchibhatla MN, Cohen HJ. Impact of anemia on mortality, cognition, and function in communitydwelling elderly. Am J Med 2006;119:327-334.

2. Patel KV, Harris TB, Faulhaber M, et al. Racial variation in the relationship of anemia with mortality and mobility disability among older adults. Blood 2007;109:4663-4670.

3. Zakai NA, Katz R, Hirsch C, et al. A prospective study of anemia status, hemoglobin concentration, and mortality in an elderly cohort: the Cardiovascular Health Study. Arch Intern Med 2005;165:2214-2220.

4. Guralnik JM, Eisenstaedt RS, Ferrucci L, Klein HG, Woodman RC. Prevalence of anemia in persons 65 years and older in the United States: evidence for a high rate of unexplained anemia. Blood 2004;104:2263-2268.

5. Salive ME, Cornoni-Huntley J, Guralnik JM, et al. Anemia and hemoglobin levels in older persons: relationship with age, gender, and health status. J Am Geriatr Soc 1992;40:489-496.

6. Beghé C, Wilson A, Ershler WB. Prevalence and outcomes of anemia in geriatrics: a systematic review of the literature. Am J Med 2004;116(suppl 7A):3S-10S.
7. Patel KV, Longo DL, Ershler WB, et al. Haemoglobin concentration and the risk of death in older adults: differences by race/ethnicity in the NHANES III follow-up. $\mathrm{Br}$ J Haematol 2009;145:514-523.

8. Atkinson HH, Cesari M, Kritchevsky SB, et al. Predictors of combined cognitive and physical decline. J Am Geriatr Soc 2005;53:1197-1202.

9. Atti AR, Palmer K, Volpato S, Zuliani G, Winblad B, Fratiglioni L. Anaemia increases the risk of dementia in cognitively intact elderly. Neurobiol Aging 2006;27:278-284.

10. Beard CM, Kokmen E, O’Brien PC, Anía BJ, Melton LJ 3rd. Risk of Alzheimer's disease among elderly patients with anemia: population-based investigations in Olmsted County, Minnesota. Ann Epidemiol 1997;7:219-224.

11. Deal JA, Carlson MC, Xue QL, Fried LP, Chaves PH. Anemia and 9-year domain-specific cognitive decline in community-dwelling older women: the Women's Health and Aging Study II. J Am Geriatr Soc 2009;57:1604-1611.

12. Faux NG, Ellis KA, Ames D, et al. Alzheimer's disease is associated with lower hemoglobin levels and anemia: the Australian Imaging Biomarker Lifestyle (AIBL) study of aging. Alzheimers Dement 2010;6:S528.

13. Lucca U, Tettamanti M, Mosconi P, et al. Association of mild anemia with cognitive, functional, mood and quality of life outcomes in the elderly: the "Health and Anemia" study. PLoS One 2008;3:e1920.

14. Shah RC, Wilson RS, Tang Y, Dong X, Murray A, Bennett DA. Relation of hemoglobin to level of cognitive function in older persons. Neuroepidemiology 2009;32: $40-46$.

15. Shah RC, Buchman AS, Wilson RS, Leurgans SE, Bennett DA. Hemoglobin level in older persons and incident Alzheimer disease: prospective cohort analysis. Neurology 2011;77:219-226.

16. Terekeci HM, Kucukardali Y, Onem Y, et al. Relationship between anaemia and cognitive functions in elderly people. Eur J Intern Med 2010;21:87-90.

17. Peters R, Burch L, Warner J, Beckett N, Poulter R, Bulpitt C. Haemoglobin, anaemia, dementia and cognitive decline in the elderly, a systematic review. BMC Geriatr 2008;8:18

18. World Health Organization. Nutritional Anemias: Reports of a WHO Scientific Group. Geneva: World Health Organisation; 1968.

19. Teng EL, Chui HC. The Modified Mini-Mental State (3MS) examination. J Clin Psychiatry 1987;48:314-318.

20. Davis TC, Long SW, Jackson RH, et al. Rapid estimate of adult literacy in medicine: a shortened screening instrument. Fam Med 1993;25:391-395.

21. Rothman R, Malone R, Bryant B, Horlen C, DeWalt D, Pignone $\mathrm{M}$. The relationship between literacy and glycemic control in a diabetes disease-management program. Diabetes Educ 2004;30:263-273.

22. Radloff L. The CES-D scale: a self-report depression scale for research in the general population. Appl Psychol Meas 1977;1:385-401.

23. Cockcroft DW, Gault MH. Prediction of creatinine clearance from serum creatinine. Nephron 1976;16:31-41.

24. Livak KJ. SNP genotyping by the $5^{\prime}$-nuclease reaction. Methods Mol Biol 2003;212:129-147.

25. Inzitari M, Studenski S, Rosano C, et al. Anemia is associated with the progression of white matter disease in older adults with high blood pressure: the cardiovascular health study. J Am Geriatr Soc 2008;56:1867-1872. 
26. Debette S, Markus HS. The clinical importance of white matter hyperintensities on brain magnetic resonance imaging: systematic review and meta-analysis. BMJ 2010;341:c3666.

27. Assaraf MI, Diaz Z, Liberman A, et al. Brain erythropoietin receptor expression in Alzheimer disease and mild cognitive impairment. J Neuropathol Exp Neurol 2007;66:389-398.

28. Hasselblatt M, Ehrenreich H, Sirén AL. The brain erythropoietin system and its potential for therapeutic exploitation in brain disease. J Neurosurg Anesthesiol 2006;18: 132-138.

29. Maiese K, Li F, Chong ZZ. New avenues of exploration for erythropoietin. JAMA 2005;293:90-95.

30. Li G, Ma R, Huang C, et al. Protective effect of erythropoietin on beta-amyloid-induced PC12 cell death through antioxidant mechanisms. Neurosci Lett 2008; 442:143-147.

31. Sun ZK, Yang HQ, Pan J, et al. Protective effects of erythropoietin on tau phosphorylation induced by betaamyloid. J Neurosci Res 2008;86:3018-3027.

32. Yavuz BB, Cankurtaran M, Haznedaroglu IC, et al. Iron deficiency can cause cognitive impairment in geriatric patients. J Nutr Health Aging 2012;16:220-224.

33. Moore E, Mander A, Ames D, Carne R, Sanders K, Watters D. Cognitive impairment and vitamin B12: a review. Int Psychogeriatr 2012;6:1-16.

34. Song X, Mitnitski A, Rockwood K. Nontraditional risk factors combine to predict Alzheimer disease and dementia. Neurology 2011;77:227-234.

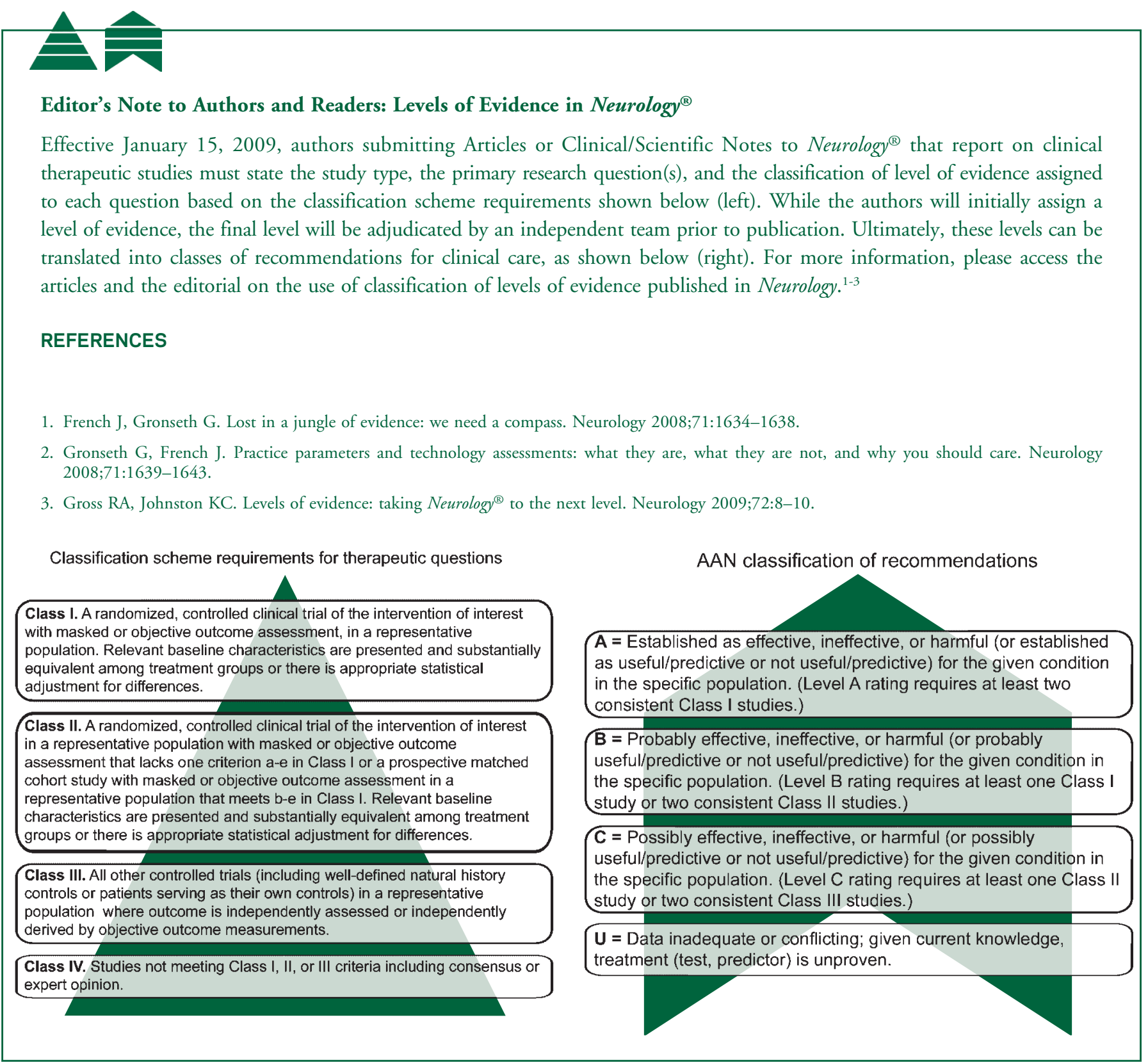

